\title{
NOUVELLE
}

\section{Rôle pathogénique de l'expression anormale de la tétraspanine CD9 par les cellules épithéliales pariétales dans les glomérulopathies extracapillaires}

Hélène Lazareth ${ }^{1-4}$, Olivia Lenoir ${ }^{1,2}$, Carole Hénique ${ }^{5}$, Cédric Bouzigues ${ }^{4}$, Claude Boucheix ${ }^{6}$, Pierre-Louis Tharaux ${ }^{1,2}$
${ }^{1}$ Inserm UMRS 970, Paris centre de recherche cardiovasculaire (PARCC), 56 rue Leblanc, 75015 Paris, France.

${ }^{2}$ Université de Paris, UMRS 970, 56 rue Leblanc, 75015 Paris, France.

${ }^{3}$ Service de néphrologie, Hôpital européen Georges-Pompidou, Assistance publique-Hôpitaux de Paris, 56 rue Leblanc, 75015 Paris, France.

${ }^{4}$ Laboratoire d'optique et biosciences, École polytechnique, CNRS UMR7645, Inserm U1182, Université Paris-Saclay, 91128 Palaiseau, France.

${ }^{5}$ Institut Mondor de recherche biomédicale, Inserm U955, équipe 21, Université Paris-est Créteil, 94010 Créteil, France. ${ }^{6}$ Inserm U935, Université Paris-Sud, 94800 Villejuif, France. helene.lazareth@aphp.fr pierre-louis.tharaux@inserm.fr
> Chaque rein humain est composé d'environ un million d'unités fonctionnelles ou néphrons, constitués chacun d'un glomérule suivi d'un tubule. Le glomérule est un système capillaire particulier, qui est le lieu de la filtration sélective du plasma sanguin pour constituer l'urine primitive. Environ $20 \%$ du plasma qui transite par les glomérules traverse la paroi du capillaire glomérulaire, et cette urine primitive est recueillie dans une chambre tapissée par un épithélium de cellules pariétales, puis évacuée par le tubule. La destruction des glomérules se traduit d'abord par une fuite de grosses protéines du plasma dans l'urine, telles que l'albumine ou les immunoglobulines et, dans les cas les plus graves, par la présence de cellules sanguines dans l'urine (hématurie microscopique). Lorsqu'un grand nombre de glomérules est détruit, la filtration du plasma est réduite et une insuffisance rénale se développe. Certaines maladies des glomérules sont dues à leur invasion destructrice par les cellules épithéliales pariétales. L'origine de ces cellules, extérieure au capillaire, a fait nommer « glomérulopathies extracapillaires »ces maladies rares mais graves. Celles-ci sont associées soit à une inflammation auto-immune du capillaire, causant une glomérulonéphrite extracapillaire
(GNEC) dite « à croissants », d'évolution rapide, soit à la perte de podocytes $^{l}$, déterminée par des causes intriquées (génétiques, toxiques, mécaniques ou métaboliques), et conduisant à la sclérose progressive du glomérule ou «hyalinose segmentaire et focale » (HSF).

La GNEC et la HSF constituent deux entités nosologiques très différentes du point de vue clinique, étiologique, évolutif, et thérapeutique. L'essentiel des travaux de recherche a porté jusqu'à présent sur l'identification et le traitement de leurs causes. Celles-ci sont identifiées dans le cas des GNEC, mais les traitements ne sont que très partiellement efficaces. Dans la plupart des cas de HSF, la cause n'est pas connue ou n'est pas encore accessible à un traitement, par exemple lorsqu'une anomalie génétique du podocyte est détectée. Pour ces deux maladies rénales, il est donc important d'élucider les mécanismes de la destruction glomérulaire, qui est associée, dans les deux cas, à l'activation des cellules épithéliales pariétales, situées en périphérie du glomérule. Ces cellules, identifiées par l'expression de la claudine 1 et des cytokératines 1 et 4 , acquièrent un phénotype de transition

${ }^{1}$ Les podocytes désignent les péricytes du capillaire glomérulaire. épithélio-mésenchymateuse, expriment la molécule d'adhérence CD44, prolifèrent et migrent de manière orientée contre le flux de plasma filtré et en direction du capillaire glomérulaire, auquel elles adhèrent. Elles forment ainsi des ponts (synéchies) entre la paroi du glomérule et le capillaire. Ces évènements conduisent à la destruction du glomérule et à l'insuffisance rénale. Dans le cas des GNEC, les cellules épithéliales pariétales constituent plus de $80 \%$ des cellules du croissant (la lésion histopathologique caractéristique des GNEC), qui contient également des cellules dérivées des podocytes, un infiltrat inflammatoire et des protéines matricielles $[1,2]$.

$\varepsilon$ n recherchant des marqueurs moléculaires exprimés à la surface des cellules épithéliales pariétales et pouvant participer au processus de destruction du glomérule, nous avons découvert que la quantité des ARNm codant la tétraspanine CD9 et celle de la protéine elle-même étaient augmentées dans les glomérules de souris modèles de GNEC nécrosante à croissants [3]. Cette surexpression de CD9, une protéine membranaire appartenant à la famille des tétraspanines, précède celle du récepteur de l'acide hyaluronique, CD44, précédemment décrit dans cette maladie glomérulaire et marqueur classique du phénotype anormal des cellules 


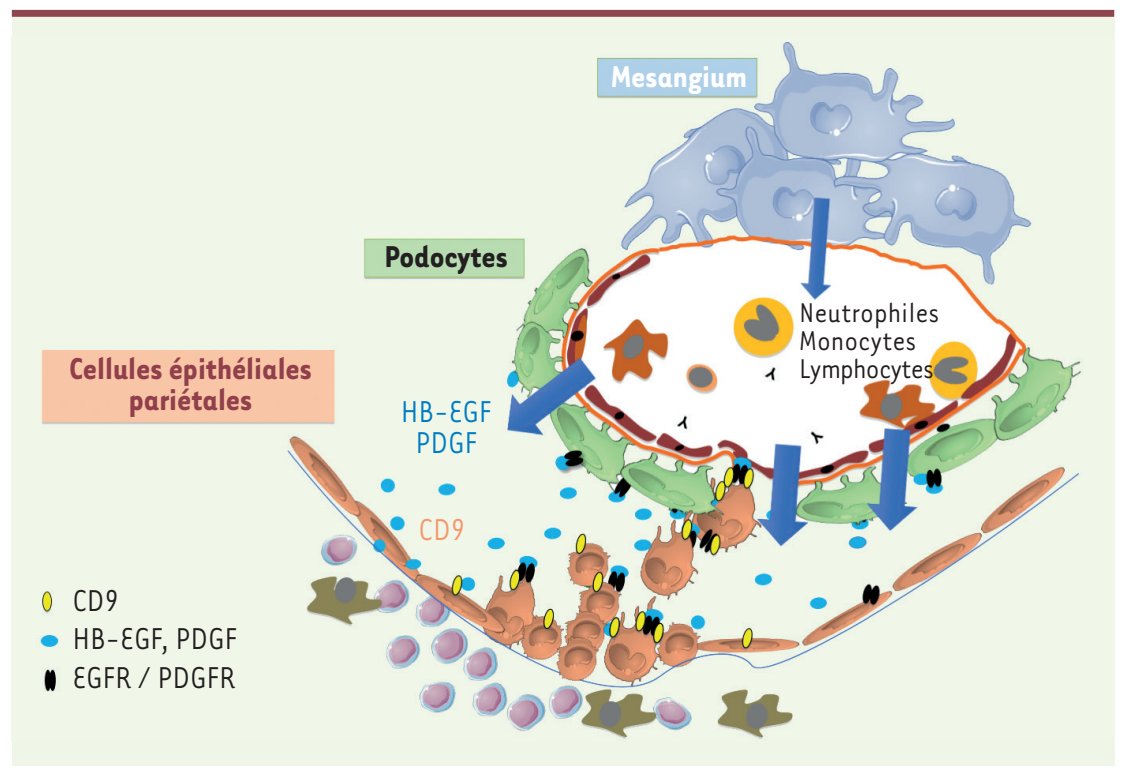

Figure 1. Recrutement pathogénique des cellules épithéliales pariétales vers le capillaire glomérulaire au cours des glomérulonéphrites extracapillaires « à croissants 》 (GNEC) et de la hyalinose segmentaire et focale (HSF). Chez l'homme et chez la souris, l'expression non physiologique de CD9 (ovale jaune) par les cellules épithéliales pariétales est un marqueur des glomérulopathies extracapillaires, qui sont caractérisées par le recrutement de cellules épithéliales pariétales anormales (en marron) vers le capillaire glomérulaire. Nous montrons que les complexes moléculaires dépendants de CD9 ajustent les niveaux de prolifération des cellules épithéliales pariétales et leur migration selon un gradient de substances chimiotactiques (comme l'HB-EGF et le PDGF-BB) provenant du capillaire glomérulaire (flèche bleue)

épithéliales pariétales [4]. Dans le rein normal, la tétraspanine CD9 est fortement exprimée par les cellules du tubule contourné distal et du tubule collecteur, mais pas par les cellules du glomérule. La fonction des tétraspanines reste débattue, mais ces protéines ont la particularité de former des complexes avec des partenaires protéiques localisés principalement dans la membrane cellulaire et de s'associer entre elles pour structurer des microdomaines membranaires [5]. Les tétraspanines contrôlent les activités des molécules qui leur sont associées par compartimentation membranaire, régulation $\mathrm{du}$ trafic (deux mécanismes particulièrement étudiés pour les couples CD81/ CD19 [6] et TspanC8/ADAM10 [a disintegrin and metalloproteinase domaincontaining protein 10] [7]), et interaction directe (CD9 et fonction juxtacrine de proHB-EGF [pro(heparin-binding epidermal growth factor-like growth factor)] [8]). Les microdomaines membranaires pourraient constituer des sites d'intégration de différents processus de signalisation intracellulaire en réponse à des stimulations extérieures. Parmi les molécules associées partiellement à ces microdomaines figurent, entre autres, le récepteur de l'EGF (epithelial growth factor receptor, EGFR), le précurseur membranaire de I'HB-EGF (heparin-binding EGF-like growth factor), des métalloprotéases comme ADAM 10 et ADAM 17, et les intégrines $\beta 1$ et $\beta 4$. Le rôle joué par ces molécules dans les glomérulopathies nous a amenés à rechercher un rôle de CD9 dans la survenue des HSF et GNEC. L'utilisation de souris mutantes dont le gène $C d 9$ était soit invalidé de manière constitutive et ubiquiste, soit invalidé spécifiquement dans certaines cellules du glomérule, a permis de déterminer l'implication des cellules épithéliales pariétales dans ces deux maladies [3].
Les souris $\mathrm{Cd}^{-/-}$, chez lesquelles le gène $C d g$ a été invalidé de façon constitutive, sont protégées de la GNEC dans le modèle d'injection de sérum néphrotoxique $[3,9]$. Comme CD9 est fortement exprimé à la surface des plaquettes sanguines et de sous-populations de lymphocytes $T$ et $B$, une invalidation conditionnelle de $C d 9$ dans les cellules progénitrices des plaquettes a permis d'exclure le rôle du CD9 plaquettaire dans la survenue de la maladie rénale. De même, une greffe de moelle osseuse provenant de souris $C d 9^{-/-}$à des souris $\mathrm{Cd}^{+/+}$ne protège pas les souris contre les effets du sérum néphrotoxique qui déclenche une GNEC. À l'inverse, les souris $\mathrm{Cd}^{-/-}$restent protégées contre ces effets après une greffe de moelle osseuse provenant d'une souris $C d 9^{+/+}$, ce qui suggère que des cellules spécifiquement rénales exprimant CD9 sont impliquées dans la survenue de la maladie. L'invalidation sélective du gène $C d 9$ dans les podocytes par le système Cre-Lox ne prévient pas la survenue de la GNEC, alors que l'invalidation sélective de $C d 9$ dans les cellules épithéliales pariétales protège de la maladie, ce qui oriente la recherche du mécanisme physiopathologique vers les cellules épithéliales pariétales. L'effet de l'invalidation de $C d 9$ dans les cellules épithéliales pariétales a également été testé sur un modèle murin de HSF induite par l'injection d'acétate de désoxycorticostérone combinée à un régime hypersalé et à une néphrectomie unilatérale. Comme pour les souris modèles de GNEC (souris

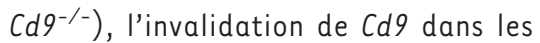
cellules épithéliales pariétales protège significativement les animaux de la HSF expérimentale.

Une surexpression de CD9 semblable à celle observée dans les modèles murins est détectée dans les biopsies rénales des patients atteints de HSF ou de GNEC, suggérant un rôle physiopathologique de CD9 chez l'homme également. Afin de mieux comprendre comment l'expression anormale de CD9 par les cellules 
épithéliales pariétales est impliquée dans le développement de ces maladies, nous avons fait l'hypothèse que CD9 pourrait influencer la réponse cellulaire à des facteurs pouvant causer la migration et la prolifération anormales de ces cellules.

Il avait été montré que certains facteurs de croissance comme I'HB-EGF et le PDGF (platelet-derived growth factor) étaient impliqués dans les glomérulopathies de la souris, avec une forte présomption de leur implication chez l'homme également [10]. Effectivement, l'inhibition à $85 \%$ de l'expression de CD9 par la technique d'interférence $A R N$ réduit considérablement l'adhérence cellulaire spontanée et la prolifération des cellules d'une lignée de cellules épithéliales pariétales induite par le PDGF-BB ou l'HB-EGF. L'interférence $A R N$ réduit également la migration des cellules épithéliales pariétales en réponse à I'HB-EGF et au PDGF-BB. La constatation que les cellules épithéliales pariétales migrent vers le capillaire glomérulaire et s'y attachent au cours des GNEC et des HSF suggère qu'elles pourraient répondre à un gradient chimiotactique produit par le capillaire endommagé. Un dispositif expérimental de canaux microfluidiques a permis de tester cette hypothèse nouvelle, et d'analyser et modéliser la migration cellulaire en présence d'un gradient de PDGF-BB, un facteur chimiotactique candidat dont l'expression est augmentée dans les capillaires inflammatoires. Ce dispositif a permis de montrer que l'extinction de l'expression de $C d 9$ n'altère pas la motilité spontanée des cellules épithéliales pariétales, mais neutralise sélectivement leur migration en réponse au gradient de PDGF-BB. La généralisation de ces expériences a révélé que les cellules épithéliales pariétales migrent selon un gradient de concentration croissant de PDGF-BB ou d'HB-EGF, et que CD9 contrôle cette migration orientée par l'activation des récepteurs de l'EGF et du PDGF [3].
L'invalidation du gène $C d 9$ chez la souris s'accompagne également d'une réduction considérable de l'expression de l'intégrine $\beta 1$, une protéine augmentée dans les lésions glomérulaires de patients atteints de HSF et de GNEC, en particulier dans les cellules épithéliales pariétales, ce qui suggère qu'elle pourrait jouer un rôle pathogénique. L'ensemble de ces observations indique que l'intégrine $\beta 1$, comme CD9, est un nouveau marqueur des cellules épithéliales pariétales pathologiques. II existe par ailleurs, dans les cellules épithéliales pariétales génétiquement déficientes en CD9, une diminution nette des voies de signalisation des récepteurs de facteurs de croissance PDGFR $\beta$ et $\varepsilon G F R$ liée à une expression réduite de ces récepteurs et à un défaut de leur phosphorylation, d'où résulte une phosphorylation réduite de la protéine FAK (focal adhesion kinase) qui participe à l'adhérence et à la migration cellulaire. Il est vraisemblable que l'atteinte de ces voies de signalisation est responsable de l'absence de migration de ces cellules génétiquement modifiées en réponse à un gradient de facteur chimiotactique [3].

Ces résultats obtenus dans des modèles animaux sont transposables à la pathologie humaine dans la mesure où l'on observe une colocalisation de CD9 avec l'intégrine $\beta 1$, principalement à la surface des cellules épithéliales pariétales, dans la GNEC avec présence d'anticorps dirigés contre le cytoplasme des polynucléaires neutrophiles (anti-neutrophil cytoplasmic antibodies, ANCA) ou associée au lupus érythémateux disséminé, ainsi que dans les HSF, alors que les glomérulopathies «non prolifératives» ne présentent pas cette caractéristique. D'autres modèles ont permis de conclure qu'une altération initiale des podocytes n'est vraisemblablement pas suffisante pour causer une activation des cellules épithéliales pariétales accompagnée de l'expression de CD9, à laquelle pourraient en revanche contribuer des facteurs endothéliaux, qui favoriseraient ainsi le développement d'une HSF [11].

L'ensemble de ces résultats permet de décrire pour la première fois un mécanisme commun expliquant le développement des lésions extra-capillaires au cours de deux maladies glomérulaires de causes très différentes (GNEC et HSF), et démontre que si ce mécanisme est inhibé, l'insuffisance rénale est beaucoup moins sévère. Cette découverte suggère que l'expression de CD9 par les cellules épithéliales pariétales dans la HSF et la GNEC contribue de manière décisive à la formation des lésions glomérulaires (synéchies, sclérose et croissants) en favorisant la migration, la prolifération et la transition épithélio-mésenchymateuse de ces cellules. Ces évènements impliquent, au moins en partie, l'amplification des voies de signalisation HB-EGF-EGFR et PDGFR, et l'augmentation d'expression de l'intégrine $\beta 1$. Il reste à déterminer l'utilité diagnostique et pronostique de la mise en évidence d'une expression anormale de $\operatorname{CD} 9$ et de l'intégrine $\beta 1$ dans les cellules épithéliales pariétales des patients atteints de ces maladies, une expression dont notre étude a révélé la précocité d'apparition et la complémentarité avec le marqueur de référence CD44.

Ces résultats invitent donc à formuler un nouveau concept physiopathologique concernant les glomérulopathies extracapillaires, GNEC et HSF : I'apparition des molécules CD9 à la surface des cellules épithéliales pariétales agit comme un amplificateur de leur sensibilité aux signaux moléculaires émis par les cellules de voisinage des glomérules atteints par la maladie. La réponse cellulaire ainsi amplifiée déclenche la migration polarisée pathologique de ces cellules, dont le rôle actif dans la destruction glomérulaire et l'insuffisance rénale avait été sous-estimé, voire ignoré. L'inhibition ciblée de la protéine CD9, la manipulation de l'expression de 
son gène dans les cellules épithéliales pariétales ou des voies de signalisation qui conduisent à sa surexpression par ces cellules, constituent autant de perspectives thérapeutiques innovantes pour ces deux maladies rénales graves. II sera par exemple intéressant d'étudier expérimentalement l'effet d'anticorps anti-CD9 sur les propriétés des cellules épithéliales pariétales, même si la perspective d'une utilisation thérapeutique de tels anticorps in vivo se heurte au problème de la distribution physiologiquement très large de CD9. Au-delà de la mise en évidence de l'implication de la protéine CD9, la connaissance des mécanismes pathogéniques dans la GNEC et la HSF demande à être approfondie (étude des signalisations coordonnées par CD9 et plus généralement, des mécanismes qui conduisent au comportement destructeur des cellules pariétales), car elle pourrait ouvrir de nouvelles perspectives thérapeutiques. $\diamond$

De novo expression of tetraspanin CD9 in parietal epithelial cells promotes extracapillary glomerulonephritis

\section{LIENS D'INTÉRÊT}

Les auteurs déclarent n'avoir aucun lien d'intérêt concernant les données publiées dans cet article.

\section{RéFÉRENCES}

1. Smeets B, Uhlig S, Fuss A, et al. Tracing the origin of glomerular extracapillary lesions from parietal epithelial cells. J Am Soc Nephrol 2009 ; 20 : 2604-15.

2. Shankland SJ, Smeets B, Pippin JW, Moeller MJ. The emergence of the glomerular parietal epithelial cell. Nat Rev Nephrol $2014 ; 10$ : 158-73.

3. Lazareth $\mathrm{H}$, Henique $\mathrm{C}$, Lenoir 0 , et al. The tetraspanin CD9 controls migration and proliferation of parietal epithelial cells and glomerular disease progression. Nat Commun $2019 ; 10: 3303$.

4. Eymael J, Sharma S, Loeven MA, et al. CD44 is required for the pathogenesis of experimental crescentic glomerulonephritis and collapsing focal segmental glomerulosclerosis. Kidney Int 2018 ; 93 : 626-42.
5. Charrin S, Jouannet S, Boucheix C, Rubinstein $\varepsilon$. Tetraspanins at a glance.J Cell Sci $2014 ; 127$ : 3641-48.

6. Levy S, Shoham T. The tetraspanin web modulates immune-signalling complexes. Nat Rev Immunol $2005 ; 5: 136-48$.

7. Saint-Pol J, Eschenbrenner $\varepsilon$, Dornier $\varepsilon$, et al. Regulation of the trafficking and the function of the metalloprotease ADAM10 by tetraspanins. Biochem Soc Trans 2017 ; 45 : 937-44.

8. Nakamura K, Iwamoto R, Mekada $\varepsilon$. Membraneanchored heparin-binding EGF-like growth factor (HB-EGF) and diphtheria toxin receptor-associated protein (DRAP27)/CD9 form a complex with integrin $\alpha 3 \beta 1$ at cell-cell contact sites. J Cell Biol 1995 ; $129: 1691-705$

9. Henique C, Bollée G, Loyer X, et al. Genetic and pharmacological inhibition of microRNA-92a maintains podocyte cell cycle quiescence and limits crescentic glomerulonephritis. Nat Commun $2017 ; 8: 1829$.

10. Bollée G, Flamant M, Schordan S, et al. Epidermal growth factor receptor promotes glomerular injury and renal failure in rapidly progressive crescentic glomerulonephritis. Nat Med 2011 ; 17 : 1242-50.

11. Luque $Y$, Lenoir $\mathrm{O}$, Bonnin $\mathrm{P}$, et al. Endothelial Epasl deficiency is sufficient to promote parietal epithelial cell activation and FSGS in experimental hypertension. J Am Soc Nephrol 2017 ; $28: 3563-78$ 